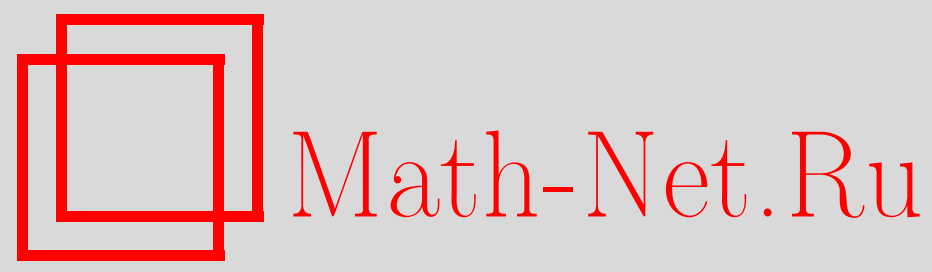

A. А. Злотник, Сун Цзян, Корректность задачи Коши для 1D уравнений вязкого теплопроводного газа с начальными данными из пространств Лебега, Матем. заметки, 2003, том 73, выпуск 5, 779-783

DOI: https://doi.org/10.4213/mzm634

Использование Общероссийского математического портала Math-Net.Ru подразумевает, что вы прочитали и согласны с пользовательским соглашением http://www.mathnet.ru/rus/agreement

Параметры загрузки:

IP : 54.198 .67 .100

26 апреля 2023 г., 18:12:10

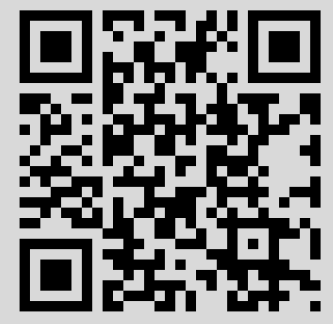




\section{КОРРЕКТНОСТЬ ЗАДАЧИ КОШИ ДЛЯ 1D УРАВНЕНИЙ ВЯЗКОГО ТЕПЛОПРОВОДНОГО ГАЗА С НАЧАЛЬНЫМИ ДАННЫМИ ИЗ ПРОСТРАНСТВ ЛЕБЕГА}

\section{А. А. Злотник, Сун Цзян}

1. Изучается задача Коши для системы квазилинейных уравнений одномерного движения вязкого теплопроводного газа [1]

$$
\begin{gathered}
\eta_{t}=v_{x}, \quad v_{t}=\sigma_{x}, \quad c_{V} \theta_{t}=\left(\kappa \rho \theta_{x}\right)_{x}+\sigma v_{x} \quad \text { в } Q, \\
\sigma=\mu \rho v_{x}-R \rho \theta, \quad \rho=1 / \eta \quad \text { в } Q, \\
\eta(x, t) \rightarrow \eta_{ \pm}, \quad v(x, t) \rightarrow v_{ \pm}, \quad \theta(x, t) \rightarrow \theta_{ \pm} \quad \text { при } \quad x \rightarrow \pm \infty, \quad 0<t<T, \\
\eta(x, 0)=\eta^{0}(x), \quad v(x, 0)=v^{0}(x), \quad \theta(x, 0)=\theta^{0}(x) \quad \text { на } \mathbb{R},
\end{gathered}
$$

где $Q \equiv Q_{T}:=\mathbb{R} \times(0, T)$. Искомые функции $\eta, v, \theta$ - удельный объем, скорость, температуразависят от лагранжевых массовых координат $(x, t)$. Функции $\sigma$ и $\rho$ - плотность и напряжение. Величины $\mu, R, c_{V}, \kappa$ - положительные физические константы, величины $\eta_{ \pm}>0, v_{ \pm}, \theta_{ \pm}$- также константы.

Глобальные сильные решения задачи (1)-(4) были изучены в работах [1]-[3] для случая начальных данных $\eta^{0}, v^{0}, \theta^{0}$ из пространства Соболева $H^{1}$.

Глобальные слабые решения соответствуюших начально-краевых задач для случая $\eta^{0}, v^{0}, \theta^{0}$ из пространств Лебега были исследованы в [4]-[9]. В настоящей работе мы переносим на задачу Коши результаты о единственности слабых решений и их непрерывной по Липшицу зависимости от начальных данных из [9] и о их существовании из [4]. Другие результаты о существовании слабых решений даны в [10], [11]; см. также [12] для малых данных.

Пусть начальные данные удовлетворяют условиям

$$
\eta^{0}>0, \quad \eta^{0},\left(\eta^{0}\right)^{-1} \in L^{\infty}(\mathbb{R}), \quad \eta^{0}-\eta_{ \pm}, v^{0}-v_{ \pm}, \theta^{0}-\theta_{ \pm} \in L^{2}\left(\mathbb{R}^{ \pm}\right),
$$

где $\mathbb{R}^{+}=(0,+\infty), \mathbb{R}^{-}=(-\infty, 0)$. Пусть $L^{q, r}\left(Q^{ \pm}\right), V_{2}\left(Q^{ \pm}\right)$- пространства с нормами [13] $\|w\|_{L^{q, r}\left(Q^{ \pm}\right)}:=\|\| w\left\|_{L^{q}\left(\mathbb{R}^{ \pm}\right)}\right\|_{L^{r}(0, T)}, q, r \in[1, \infty],\|w\|_{V_{2}\left(Q^{ \pm}\right)}:=\|w\|_{L^{2, \infty}\left(Q^{ \pm}\right)}+\left\|w_{x}\right\|_{L^{2}\left(Q^{ \pm}\right)}$, где $Q^{ \pm}:=\mathbb{R}^{ \pm} \times(0, T)$.

Для задачи (1)-(4) введем слабие решения $(\eta, v, \theta)$ из класса $\mathscr{A}$ :

$\eta>0, \quad \eta, \eta^{-1} \in L^{\infty}(Q), \quad \eta-\eta_{ \pm} \in L^{2, \infty}\left(Q^{ \pm}\right), \quad \eta_{t} \in L^{2}(Q), \quad v-v_{ \pm}, \theta-\theta_{ \pm} \in V_{2}\left(Q^{ \pm}\right)$.

Они удовлетворяют уравнениям $\eta_{t}=v_{x}$ и (2), интегральным тождествам

$$
\begin{gathered}
\int_{Q}\left(-v \varphi_{t}+\sigma \varphi_{x}\right) d x d t=\left.\int_{\mathbb{R}} v^{0} \varphi\right|_{t=0} d x \quad \forall \varphi \in C^{1}(\bar{Q}) \\
\int_{Q}\left(-c_{V} \theta \varphi_{t}+\kappa \rho \theta_{x} \varphi_{x}-\sigma v_{x} \varphi\right) d x d t=\left.\int_{\mathbb{R}} c_{V} \theta^{0} \varphi\right|_{t=0} d x \quad \forall \varphi \in C^{1}(\bar{Q}),
\end{gathered}
$$

где $\operatorname{supp} \varphi$ компактен в $\mathbb{R} \times[0, T)$, и начальному условию $\left.\eta\right|_{t=0}=\eta^{0}$.

Сформулируем основные результаты работы. Ниже $N>1, \bar{N}>1$ - параметры, а величины $K(N), K(\bar{N})$ не зависят от $\eta^{0}, v^{0}, \theta^{0}$.

Работа первого автора вьполнена при поддержке Российского фонда фундаментальных исследований, грант № 00-01-00207, работа второго автора вьполнена при поддержке the Special Funds for MSBRP, № G1999032801, the NNSF и the CAEP Китая. 
Tеорема 1. Слабые решения из класса $\mathscr{A}$ задачи Коши (1)-(4) единственны.

Более того, пусть $\ell=1,2 u\left(\eta^{(\ell)}, v^{(\ell)}, \theta^{(\ell)}\right)$ - решение, отвечающее начальным данным $\left(\eta^{0,(\ell)}, v^{0,(\ell)}, \theta^{0,(\ell)}\right)$, удовлетворяющим условиям (5), причем

$$
\bar{N}^{-1} \leqslant \eta^{(\ell)} \leqslant \bar{N} \quad \text { e } \quad Q, \quad\left\|v^{(\ell)}-v_{ \pm}\right\|_{V_{2}\left(Q^{ \pm}\right)}+\left\|\theta^{(\ell)}-\theta_{ \pm}\right\|_{V_{2}\left(Q^{ \pm}\right)} \leqslant \bar{N}
$$

с каким-либо $\bar{N}>1$. Тогда справедлива оченка разности решений

$$
\begin{gathered}
\|\Delta \eta\|_{L^{\infty}(Q) \cap L^{2, \infty}(Q)}+\|\Delta v\|_{V_{2}(Q)}+\|\Delta \theta\|_{L^{q_{0}, r_{0}(Q)}}+\|\Delta \theta x\|_{L^{2, r_{1}(Q)}} \\
\leqslant K(\bar{N})\left(\left\|\Delta \eta^{0}\right\|_{L^{\infty}(\mathbb{R}) \cap L^{2}(\mathbb{R})}+\left\|\Delta v^{0}\right\|_{L^{2}(\mathbb{R})}+\left\|\Delta \theta^{0}\right\|_{L^{2}(\mathbb{R})}\right)
\end{gathered}
$$

$c \bumpeq 10 б$ ми

$$
q_{0} \in[2, \infty], \quad r_{0} \in[1, \infty], \quad \frac{1}{2 q_{0}}+\frac{1}{r_{0}}=\frac{1+\varepsilon_{0}}{2}, \quad r_{1}=\frac{4}{3+\varepsilon_{0}} \quad \partial \Omega_{\text {s }} \quad \varepsilon_{0} \in\left(0, \frac{1}{2}\right] .
$$

Здесь и ниже $\Delta \eta=\eta^{(1)}-\eta^{(2)}, \Delta \eta^{0}=\eta^{0,(1)}-\eta^{0,(2)} u$ m.д.

Отметим, что в силу условий теоремы $\Delta \eta^{0}, \Delta v^{0}, \Delta \theta^{0} \in L^{2}(\mathbb{R})$ и $\Delta \eta \in L^{2, \infty}(Q), \Delta v, \Delta \theta \in$ $V_{2}(Q)$; как следствие, $\Delta \theta \in L^{q_{0}, r_{0}}(Q)$.

ТЕОРема 2. Пусть $\eta_{ \pm}>0, v_{-} \leqslant v_{+}, \theta_{ \pm}=\theta_{\infty}>0$. Пусть выполнены условия (5), а maжxс $N^{-1} \leqslant \eta^{0} \leqslant N, N^{-1} \leqslant \theta^{0} u$

$$
\left\|\eta^{0}-\eta_{ \pm}\right\|_{L^{2}\left(\mathbb{R}^{ \pm}\right)}+\left\|v^{0}-v_{ \pm}\right\|_{L^{2}\left(\mathbb{R}^{ \pm}\right) \cap L^{4}\left(\mathbb{R}^{ \pm}\right)}+\left\|\theta^{0}-\theta_{\infty}\right\|_{L^{2}(\mathbb{R})} \leqslant N
$$

с каким-либо $N>1$. Тогда существует слабое решение $(\eta, v, \theta)$ из класса $\mathscr{A}$ задачи Коши (1)-(4), удовлетворяющее оченкам

$$
\begin{gathered}
K(N)^{-1} \leqslant \eta \leqslant K(N), \quad K(N)^{-1} \leqslant \theta \quad \text { e } \quad Q, \\
\left\|\eta-\eta_{ \pm}\right\|_{L^{2, \infty}\left(Q^{ \pm}\right)}+\left\|\eta_{t}\right\|_{L^{2}(Q)}+\left\|v-v_{ \pm}\right\|_{V_{2}\left(Q^{ \pm}\right)}+\left\|\theta-\theta_{\infty}\right\|_{V_{2}(Q)} \leqslant K(N), \\
\left\|v-v_{ \pm}\right\|_{L^{4, \infty}\left(Q^{ \pm}\right)}+\left\|v v_{x}\right\|_{L^{2}(Q)} \leqslant K(N) .
\end{gathered}
$$

2. Доказательство теоремы 1 опирается на результаты о свойствах слабых решений задачи Коши для линейных параболических уравнений с негладкими данньми. Эти решения можно понимать аналогично (6), (7).

Рассмотрим следующую задачу Коши:

$$
(\alpha u)_{t}=\left(\beta u_{x}-\psi\right)_{x}+f \quad \text { в } Q, \quad u(x, t) \rightarrow 0 \quad \text { при } \quad|x| \rightarrow \infty, \quad 0<t<T,\left.\quad u\right|_{t=0}=u^{0}
$$

с коэффициентами $\alpha$ и $\beta$ такими, что $\alpha, \beta \in L^{\infty}(Q), N^{-1} \leqslant \alpha \leqslant N, N^{-1} \leqslant \beta \leqslant N,\left\|\alpha_{t}\right\|_{L^{2,4 / 3}(Q)}$ $\leqslant N$. Пусть для краткости $\|\cdot\|_{L^{p}}:=\|\cdot\|_{L^{p}(\mathbb{R})}, 1 \leqslant p \leqslant \infty$.

ПРЕДЛОЖЕнИЕ 1. Пусть $u^{0} \in L^{2}(\mathbb{R}), \psi \in L^{2}(Q), f \in L^{q, r}(Q)$ с некоторыми $q \in[1,2]$, $r \in[1,4 / 3], 1 /(2 q)+1 / r=5 / 4$. Тогда существует единственное слабое решение и из класса $V_{2}(Q)$ задачи (9), причем

$$
\|u\|_{V_{2}(Q)} \leqslant K(N)\left(\left\|u^{0}\right\|_{L^{2}}+\|\psi\|_{L^{2}(Q)}+\|f\|_{L^{q, r}(Q)}\right) .
$$

Наряду с (9) рассмотрим также несколько иную задачу:

$$
\bar{\alpha} w_{t}=\left(\bar{\beta} w_{x}-\bar{\psi}\right)_{x}+\bar{f} \quad \text { в } \quad Q, \quad w(x, t) \rightarrow 0 \quad \text { при } \quad|x| \rightarrow \infty, \quad 0<t<T,\left.\quad w\right|_{t=0}=w^{0}
$$

с коэффициентами $\bar{\alpha}, \bar{\beta}$ такими, что $\bar{\alpha}, \bar{\beta} \in L^{\infty}(Q), N^{-1} \leqslant \bar{\alpha} \leqslant N, N^{-1} \leqslant \bar{\beta} \leqslant N,\left\|\bar{\beta}_{t}\right\|_{L^{2,4 / 3}(Q)}$ $\leqslant N$. Введем пространство $W(Q)$ с нормой $\|w\|_{W(Q)}:=\|w\|_{L^{2}(Q)}+\left\|w_{x}\right\|_{L^{2, \infty}(Q)}+\left\|w_{t}\right\|_{L^{2}(Q)}$. Положим также $\left(I_{0} y\right)(t)=\int_{0}^{t} y(\tau) d \tau$.

Между задачами (9) и (10) существует тесная связь. 
ПРеДлОЖенИЕ 2. 1. Пусть выполнены условия предложсения 1 и $I_{0} f \in L^{2, \infty}(Q)$. Тогда функиия $w=w^{0}+I_{0}\left(\beta u_{x}-\psi\right)$ с $w^{0} \in H^{1}(\mathbb{R})$ является слабым решением из класса $W(Q)$ задачи (10) с данными

$$
\bar{\alpha}=\beta^{-1}, \quad \bar{\beta}=\alpha^{-1}, \quad \bar{\psi}=-\alpha^{-1}\left(\left.\alpha\right|_{t=0} u^{0}-w_{x}^{0}+I_{0} f\right), \quad \bar{f}=-\beta^{-1} \psi .
$$

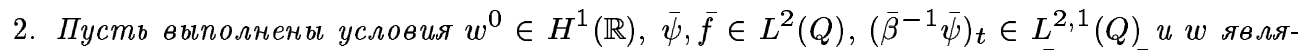
ется слабым решением из класса $W(Q)$ задачи (10). Тогда функиия $u=\bar{\beta} w_{x}-\bar{\psi}$ является слабым решением из класса $V_{2}(Q)$ задачи (9) с данными

$$
\alpha=\bar{\beta}^{-1}, \quad \beta=\bar{\alpha}^{-1}, \quad \psi=-\bar{\alpha}^{-1} \bar{f}, \quad f=-\left(\bar{\beta}^{-1} \bar{\psi}\right)_{t}, \quad u^{0}=\left.\bar{\beta}\right|_{t=0} w_{x}^{0}-\left.\bar{\psi}\right|_{t=0} .
$$

СледСтвиЕ 1. В условиях на данные из предложсения $2, n$. 2 существует единственное слабое решение из класса $W(Q)$ задачи (10), причем

$$
\|w\|_{W(Q)} \leqslant K(N)\left(\left\|w^{0}\right\|_{H^{1}(\mathbb{R})}+\left\|\left.\bar{\psi}\right|_{t=0}\right\|_{L^{2}}+\left\|\left(\bar{\beta}^{-1} \bar{\psi}\right)_{t}\right\|_{L^{2,1}(Q)}+\|\bar{f}\|_{L^{2}(Q)}\right) .
$$

ПРЕДЛОЖЕНИЕ 3. Пусть выполнены условия предложения 2, n. 2 и

$$
\left\|\bar{\alpha}_{t}\right\|_{L^{2, r_{\alpha}(Q)}} \leqslant N \quad c \quad r_{\alpha}=\frac{4}{3}\left[\left(1-\frac{2 \varepsilon_{\alpha}}{3}\right)\right]^{-1} \quad \text { для некоторого } \varepsilon_{\alpha} \in\left(0, \frac{1}{2}\right] .
$$

Тогда

$$
\sup _{\bar{Q}}|w| \leqslant K(N)\left(\left\|w^{0}\right\|_{L^{\infty} \cap L^{2}}+\|\bar{\psi}\|_{L^{2, r_{1}^{\prime}}{ }_{(Q)}}+\|\bar{f}\|_{L^{q_{0}^{\prime}, r_{0}^{\prime}(Q)}}\right)
$$

для всех $q_{0}, r_{0}, r_{1}$, удовлетворяющих (8) $c 0<\varepsilon_{0} \leqslant \varepsilon_{\alpha}$.

ПРЕДЛОЖЕнИЕ 4. Пусть выполнены условия (11) с $\bar{\alpha}=\alpha u \beta_{t} \in L^{2,4 / 3}(Q)$. Пусть также $u^{0} \in L^{1}(\mathbb{R}), \psi=0, f \in L^{1}(Q)$. Тогда существует единственное слабое решение из класса $u \in L^{2}(Q), u_{x} \in L^{2,1}(Q)$ задачи (9), причем

$$
\|u\|_{L^{q_{0}, r_{0}(Q)}}+\left\|u_{x}\right\|_{L^{2, r_{1}(Q)}} \leqslant K(N)\left(\left\|u^{0}\right\|_{L^{1}}+\|f\|_{L^{1}(Q)}\right)
$$

для всех $q_{0}, r_{0}, r_{1}$, удовлетворяющих (8) $с 0<\varepsilon_{0} \leqslant \varepsilon_{\alpha}$.

Перечисленные результаты выводятся последовательно по аналогии с соответствующими результатами для начально-краевых задач из [13], [14], [8].

3. Ниже в разделах $3,4 I=\left(x_{0}, x_{1}\right)$ - любой конечньй интервал, $|I|-$ его длина. Для обоснования теоремы 1 используется схема рассуждений из [9].

Лемма 1. Справедливъ формуль

$$
\begin{array}{ccc}
\eta=a\left(\eta^{0}+\mu^{-1} R I_{0}\left(a^{-1} \theta\right)\right) & c & a:=\exp \left(\mu^{-1} I_{0} \sigma\right), \\
I_{0} \sigma=A_{I}\left(v-v^{0}\right)+I_{0}\langle\sigma\rangle_{I} & \text { на } \quad I_{T}:=I \times(0, T),
\end{array}
$$

əде $A_{I} w(x):=\int_{x_{0}}^{x} w(\xi) d \xi-\left\langle\int_{x_{0}}^{x} w(\xi) d \xi\right\rangle_{I},\langle w\rangle_{I}:=\frac{1}{|I|} \int_{I} w(x) d x$.

Кроме того, $I_{0} \sigma \in C(\bar{Q})$ и верны оченки

$$
\|\theta\|_{L^{\infty, 4}(Q)} \leqslant K(\bar{N}), \quad \sup _{\bar{Q}}\left|I_{0} \sigma\right| \leqslant K(\bar{N}) .
$$

При помощи леммы 1 и предложений 1, 4 последовательно вьводятся следующие равномерные относительно $0<t \leqslant T$ оценки:

$$
\begin{aligned}
\|\Delta \eta\|_{L^{\infty}\left(I_{t}\right)} \leqslant & K(\bar{N})\left(\left\|\Delta \eta^{0}\right\|_{L^{\infty}(I)}+\left\|A_{I} \Delta v^{0}\right\|_{L^{\infty}(I)}+\left\|A_{I} \Delta v\right\|_{L^{\infty}\left(I_{t}\right)}\right. \\
& \left.+\left\|\Delta v_{x}\right\|_{L^{1}\left(I_{t}\right)}+\|\Delta \theta\|_{L^{\infty, 1}\left(I_{t}\right)}\right)
\end{aligned}
$$


где $K(\bar{N})$ зависит от $|I|$, но не от $I$;

$$
\begin{aligned}
\|\Delta \eta\|_{L^{\infty}\left(Q_{t}\right)} & \leqslant K(\bar{N})\left(\left\|\Delta \eta^{0}\right\|_{L^{\infty}}+\left\|\Delta v^{0}\right\|_{L^{2}}+\|\Delta v\|_{V_{2}\left(Q_{t}\right)}+\|\Delta \theta\|_{L^{\infty, 1}\left(Q_{t}\right)}\right) \\
\|\Delta v\|_{V_{2}\left(Q_{t}\right)} & \leqslant K(\bar{N})\left(\left\|\Delta v^{0}\right\|_{L^{2}}+\|d\| \Delta \eta\left\|_{L^{\infty} \cap L^{2}}\right\|_{L^{2}(0, t)}+\|\Delta \theta\|_{L^{2}\left(Q_{t}\right)}\right)
\end{aligned}
$$

где функция $d=d(t)$ такова, что $\|d\|_{L^{2}(0, T)} \leqslant K(\bar{N})$;

$$
\begin{gathered}
\|\Delta \eta\|_{L^{\infty}\left(Q_{t}\right) \cap L^{2, \infty}\left(Q_{t}\right)}+\|\Delta v\|_{V_{2}\left(Q_{t}\right)} \\
\leqslant K(\bar{N})\left(\left\|\Delta \eta^{0}\right\|_{L^{\infty} \cap L^{2}}+\left\|\Delta v^{0}\right\|_{L^{2}}+\|\Delta \theta\|_{L^{\infty, 1}\left(Q_{t}\right) \cap L^{2}\left(Q_{t}\right)}\right), \\
\|\Delta \theta\|_{L^{q_{0}, r_{0}\left(Q_{t}\right)}}+\|\Delta \theta x\|_{L^{2, r_{1}\left(Q_{t}\right)}} \\
\leqslant K(\bar{N})\left(\left\|\Delta \theta^{0}\right\|_{L^{2}}+\|\Delta \eta\|_{L^{\infty}\left(Q_{t}\right) \cap L^{2, \infty}\left(Q_{t}\right)}+\left\|\Delta v_{x}\right\|_{L^{2}\left(Q_{t}\right)}+\|\Delta \theta\|_{L^{2}\left(Q_{t}\right)}\right),
\end{gathered}
$$

для любых $q_{0}, r_{0}, r_{1}$, удовлетворяющих (8).

Теорему 1 нетрудно вывести из последних двух оценок по аналогии с [9].

4. Для доказательства теоремы 2 введем семейство усредненных начальных данных $\eta_{\varepsilon}^{0}, v_{\varepsilon}^{0}, \theta_{\varepsilon}^{0} \mathrm{c}$ параметром усреднения $\varepsilon \rightarrow 0$ и соответствующее семейство глобальных сильных решений $\left(\eta^{\varepsilon}, v^{\varepsilon}\right.$, $\left.\theta^{\varepsilon}\right)$ со свойствами [1]-[3]

$$
\begin{gathered}
\eta^{\varepsilon}-\eta_{ \pm}, v^{\varepsilon}-v_{ \pm}, \theta^{\varepsilon}-\theta_{\infty}, \eta_{x}^{\varepsilon} \in L^{2, \infty}\left(Q^{ \pm}\right), \quad v_{x}^{\varepsilon}, \theta_{x}^{\varepsilon} \in V_{2}(Q) \\
\eta_{t}^{\varepsilon}, \eta_{x t}^{\varepsilon}, v_{t}^{\varepsilon}, \theta_{t}^{\varepsilon} \in L^{2}(Q), \quad \inf _{\bar{Q}} \eta^{\varepsilon}>0, \quad \inf _{\bar{Q}} \theta^{\varepsilon}>0
\end{gathered}
$$

Эти решения удовлетворяют равномерным по $\varepsilon$ оценкам [1]-[3], [11]

$$
K^{-1} \leqslant \eta^{\varepsilon} \leqslant K, \quad K^{-1} \leqslant \theta^{\varepsilon}, \quad\left\|\eta^{\varepsilon}-\eta_{ \pm}\right\|_{L^{2, \infty}\left(Q^{ \pm}\right)}+\left\|\eta_{t}^{\varepsilon}\right\|_{L^{2}(Q)}+\left\|v^{\varepsilon}-v_{ \pm}\right\|_{V_{2}\left(Q^{ \pm}\right)} \leqslant K
$$

(здесь и ниже $K$ не зависит от $\varepsilon$ ). Пусть $\sigma^{\varepsilon}=\mu \rho^{\varepsilon} v_{x}^{\varepsilon}-R \rho^{\varepsilon} \theta^{\varepsilon}, \rho^{\varepsilon}=1 / \eta^{\varepsilon}$.

Следующие два результата имеют аналоги в [1] и [5].

ПРЕДЛОЖЕНИЕ 5. Справедлива оценка

$$
\left\|v^{\varepsilon}-v_{ \pm}\right\|_{L^{4, \infty}\left(Q_{T}^{ \pm}\right)}+\left\|v^{\varepsilon} v_{x}^{\varepsilon}\right\|_{L^{2}(Q)}+\left\|\theta^{\varepsilon}-\theta_{\infty}\right\|_{V_{2}(Q)} \leqslant K
$$

ПРЕДЛОЖЕНИЕ 6. Справедлива оченка

$$
\left\|\Delta_{h} \eta^{\varepsilon}\right\|_{L^{p, \infty}\left(I_{T}\right)} \leqslant K\left(\left\|\Delta_{h} \eta^{0}\right\|_{L^{p}(I)}+|h|^{1 / 2+1 / p}\right), \quad 2 \leqslant p \leqslant \infty
$$

при всех $h$, причем $K$ не зависит от $I, h$. В ней $\Delta_{h} f(x):=f(x+h)-f(x)$, a $I$ произвольный (возможно, бесконечный) интервал.

Указанные оценки позволяют извлечь из $\left(\eta^{\varepsilon}, v^{\varepsilon}, \theta^{\varepsilon}\right)$ последовательность (за которой мы сохраняем прежнее обозначение) такую, что при $\varepsilon \rightarrow 0$

$$
\begin{aligned}
& \eta^{\varepsilon} \rightarrow \eta \quad * \text {-слабо в } L^{\infty}(Q), \quad v^{\varepsilon}-v_{ \pm} \rightarrow v-v_{ \pm} \quad \text { *-слабо в } L^{\infty}\left(0, T ; L^{4}\left(\mathbb{R}^{ \pm}\right)\right) \text {, } \\
& \left(\eta^{\varepsilon}-\eta_{ \pm}, v^{\varepsilon}-v_{ \pm}, \theta^{\varepsilon}-\theta_{\infty}\right) \rightarrow\left(\eta-\eta_{ \pm}, v-v_{ \pm}, \theta-\theta_{\infty}\right) \quad * \text {-слабо в } \\
& L^{\infty}\left(0, T ; L^{2}\left(\mathbb{R}^{ \pm}\right)\right) \\
& \left(\eta_{t}^{\varepsilon}, v_{x}^{\varepsilon}, \theta_{x}^{\varepsilon}\right) \rightarrow\left(\eta_{t}, v_{x}, \theta_{x}\right) \quad \text { и } \quad v^{\varepsilon} v_{x}^{\varepsilon} \rightarrow w \quad \text { слабо в } L^{2}(Q), \\
& \eta^{\varepsilon} \rightarrow \eta \quad \text { и } \quad \rho^{\varepsilon} \rightarrow \rho \quad \text { сильно в } L^{p}\left(I_{T}\right), \quad 1 \leqslant p<\infty \text {. }
\end{aligned}
$$

Функция $(\eta, v, \theta)$ удовлетворяет оценкам теоремы 2 (но с $w$ в роли $v v_{x}$ ). 
Как следствие, $\rho^{\varepsilon} v^{\varepsilon} \rightarrow \rho v$ слабо в $L^{p}\left(I_{T}\right), 1 \leqslant p<4$, а $\sigma^{\varepsilon} \rightarrow \sigma$ и $\rho^{\varepsilon} \theta_{x}^{\varepsilon} \rightarrow \rho \theta_{x}$ слабо в $L^{p}\left(I_{T}\right)$, $1 \leqslant p<2$. С учетом уравнений задачи верны также оценки

$$
\left\|v_{t}^{\varepsilon}\right\|_{L^{2}\left(0, T ; H^{-1}(I)\right)} \leqslant K, \quad\left\|\theta_{t}^{\varepsilon}\right\|_{L^{1}\left(0, T ; H^{-1}(I)\right)} \leqslant K, \quad\left\|\left(\eta^{\varepsilon} \sigma^{\varepsilon}\right)_{t}\right\|_{L^{1}\left(0, T ; H^{-2}(I)\right)} \leqslant K .
$$

Поскольку и $\left\|\Delta_{h} v^{\varepsilon}\right\|_{L^{2}(Q)} \leqslant K|h|,\left\|\Delta_{h}\left(\rho^{\varepsilon} v^{\varepsilon}\right)\right\|_{L^{2}(Q)} \leqslant K\left(\left\|\Delta_{h} \eta^{0}\right\|_{L^{2}}+|h|\right), \sigma^{\varepsilon} v^{\varepsilon}=\eta^{\varepsilon} \sigma^{\varepsilon} \cdot \rho^{\varepsilon} v^{\varepsilon}$, в силу леммы 5.1 из [15] для квадратичных членов имеем

$$
\left(v^{\varepsilon}\right)^{2} \rightarrow v^{2} \text { и } v^{\varepsilon} v_{x}^{\varepsilon} \rightarrow v v_{x}=w \quad \text { слабо в } L^{2}\left(I_{T}\right), \quad \sigma^{\varepsilon} v^{\varepsilon} \rightarrow \sigma v \quad \text { слабо в } L^{p}\left(I_{T}\right),
$$

где $1 \leqslant p<4 / 3$. Полученные предельные соотношения позволяют обосновать то, что $(\eta, v, \theta)-$ слабое решение из класса $\mathscr{A}$ задачи (1)-(4) (при этом, как и в [5], в дополнение к (7) надо использовать слабую форму уравнения энергии).

\section{СПИСОК ЦИТИРОВАННОЙ ЛИТЕРАТУРЫ}

1. Антонцев С. Н., Кажихов А. В., Монахов В.Н. Краевые задачи механики неоднородной жидкости. Новосибирск: Наука, 1983. 2. Кажихов А. В. // Сиб. матем. ж. 1982. Т. 23. №1. С. 44-49. 3. Смагулов Ш. С., Дурмагамбетов А. А., Искендерова Д. А. // Дифференц. уравнения. 1993. Т. 29. №2. С. 337-348. 4. Амосов А. А., Злотник А. А. // Докл. AH CCCP. 1988. Т. 301. №1. С. 11-15. 5. Амосов А. А., Злотник А. А. // Матем. заметки. 1992. Т. 52. № 1. С. 3-16. 6. Fujita-Yashima H., Padula M., Novotný A. // Ricerche di Matematica. 1993. V. 42. № 2. Р. 199-248. 7. Амосов А. А., Злотник А. А. // Изв. вузов. Матем. 1997. Т. 41. № 4. С. 3-19. 8. Злотник А. А., Амосов А. А. // Сиб. матем. ж. 1997. Т. 38. № 4. С. 767-789. 9. Злотник А. А., Амосов А. А. // Матем. заметки. 1998. Т. 63 . №6. C. 835-846. 10. Serre D. // C. R. Acad. Sci. Paris. Sér. I. 1986. V. 303. № 14. P. 703-706. 11. Jiang S., Zhang P. // Quart. Appl. Math. (to appear).. 12. Hoff D. // J. Diff. Equations. 1992. V. 95. № 1. Р. 33-74. 13. Ладыженская О. А., Солонников В. А., Уральцева Н. Н. Линейные и квазилинейные уравнения параболического типа. М.: Наука, 1967. 14. Амосов А. А., Злотник А. А. // Дифференц. уравнения. 1997. Т. 33. №1. С. 83-95. 15. Lions P. L. Mathematical Topics in Fluid Mechanics. V. 2. Compressible Models. Oxford: Clarendon Press, 1998.

Московский энергетический институт (технический университет) 